\title{
IPTG-Inducible Episomal Expression System for Exogenous Genes in Primate Cells
}

BioTechniques 28:577-581 (March 2000)

\author{
Yanzhuang Li and Lester $\mathrm{F}$. \\ Lau \\ University of Illinois College of \\ Medicine, Chicago, IL, USA
}

\section{INTRODUCTION}

The ability to express an exogenous gene in an inducible manner in mammalian cells is often desirable, especially when the gene product is potentially cytotoxic or growth-inhibitory. To this end, several inducible expression systems have been established. Among them is the isopropyl $\beta$-D-thiogalactopyranoside (IPTG)-inducible expression system, which consists of an expression vector with a basal promoter linked to the Escherichia coli lac operator sequences (3). Genes cloned under the control of this inducible promoter can be specifically activated by the IPTG-dependent transactivator LAP267 , a chimeric protein containing the herpes simplex virus virion protein 16 (VP16) transactivation domain linked to the E. coli lac repressor (3). LAP267 functions as a temperature-sensitive transactivator, being active at $32^{\circ} \mathrm{C}$ but inactive at the normal cell growth temperature of $37^{\circ} \mathrm{C} \mathrm{(3).} \mathrm{However,} \mathrm{its} \mathrm{activ-}$ ity is restored by the presence of IPTG at the nonpermissive temperature (3).

Recently, this IPTG-inducible expression system has been modified, and its background level of expression was reduced (17). A modified mouse mammary tumor virus (MMTV) basal promoter was put in place of the simian virus 40 (SV40) early promoter used in the original vector. Two Oct-1 binding sites within the basal MMTV promoter were destroyed by mutation, resulting in very low basal or leaky expression from the inducible promoter (15). This vector system is efficacious for inducible gene expression in transfected mammalian cells (16).

We have sought to improve this inducible vector system further by incorporating the advantages of an episomal vector. The presence of the EpsteinBarr virus (EBV) origin for plasmid replication (oriP) and the EBV nuclear antigen 1 (EBNA 1) gene on the same DNA molecule allows for extra-chromosomal replication in a variety of established primate cells, but not in rodent cells (20). Such EBV-derived plasmids replicate only once per cell cycle and are not amplified on entry into cells (19); they are maintained at anywhere from a few copies to about one hundred copies per cell under selection in different cell lines, and are lost from the cell population at rates of about $5 \%$ per generation in the absence of selection $(19,20)$.

Episomal vectors such as those derived from the EBV replicon have several advantages $(8,11,12,14)$. First, the absence of integration into host-cell chromosomes obviates potential problems of integration position effects on gene expression and reduces the likelihood of any rearrangement of the transfected DNA $(8,11,12,14)$. Second, the expression levels of transfected genes are consistently higher than expression from non-episomal vectors (11). Third, recovery of the transfected episomal plasmids is simple and efficient using the Hirt's extraction procedure $(4,9)$.

Here, we report the construction of an IPTG-inducible episomal expression vector, pEpiLac, which combines the advantages of both the IPTG-inducible expression system and the EBV-based, extra-chromosomal replication system. 
We also introduced the LAP267 transactivator into the human breast carcinoma cell line MCF7, thus establishing a MCF7-derived cell line in which the pEpiLac vector can efficiently express exogenous genes in an inducible manner.

\section{MATERIALS AND METHODS}

\section{Cell Culture}

MCF7 cells and MCF7/LAP5 cells were maintained at $37^{\circ} \mathrm{C}$ and $5 \% \mathrm{CO}_{2}$ in MEM containing nonessential amino acids, plus $1 \mathrm{mM}$ sodium pyruvate, $1 \mathrm{mM}$ glutamine (all from Life Technologies, Gaithersburg, MD, USA), $10 \%$ fetal bovine serum (Intergen, Purchase, NY, USA) and 10 $\mu \mathrm{g} / \mathrm{mL}$ bovine insulin (Life Technologies). Cells were refed every $2-3$ days. Where indicated, hygromycin B (Roche Molecular Biochemicals, Indianapolis, IN, USA) or G418 (Life Technologies) was added into the medium to the final concentrations of $75 \mu \mathrm{g} / \mathrm{mL}$ or $600 \mu \mathrm{g} / \mathrm{mL}$, respectively. No G418 selection pressure is required to maintain MCF7/LAP5 cells.

\section{Transfections}

For electroporation, $2 \times 10^{7}$ cells in $0.8 \mathrm{~mL}$ PBS, $\mathrm{pH} 7.35$, and $20 \mu \mathrm{g}$ DNA were incubated at room temperature for $10 \mathrm{~min}$, electrically pulsed in a $0.4 \mathrm{~cm}$ cuvette (Bio-Rad Laboratories, Hercules, CA, USA) at $960 \mu \mathrm{FD}$ and 330 V. After 5 min incubation at room tem-

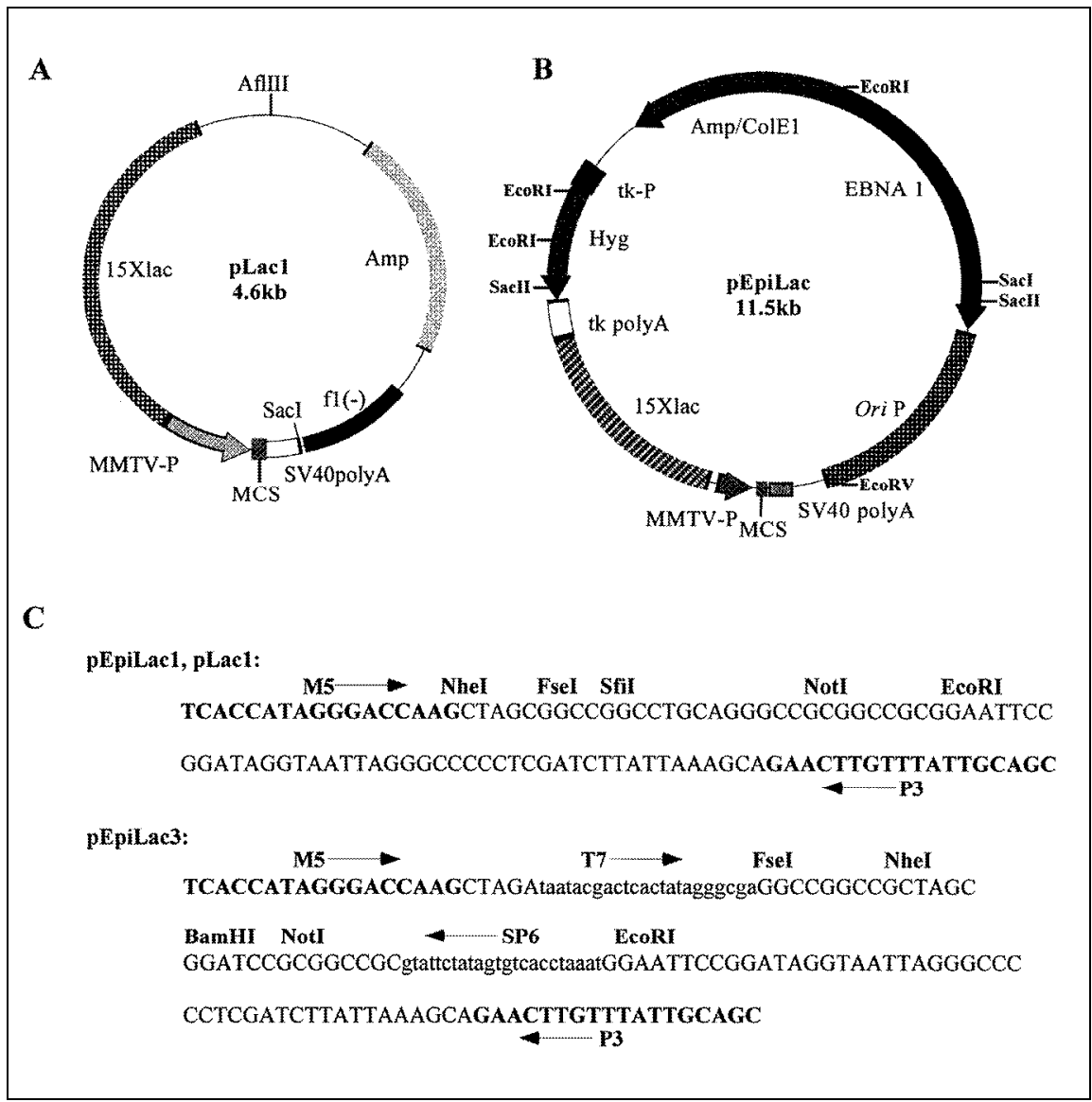

Figure 1. Schematic representation of IPTG-inducible expression vectors. The physical maps of the pLac1 non-episomal vector (A) and the pEpiLac episomal vecctor (B) are shown. Sequence of their MCS are shown in panel C. Abbreviations are: OriP, origin for plasmid replication of EB virus; EBNA1, EB virus nuclear antigen 1; Hyg, hygromycin B resistant gene; lac, lactose operator sequences; MMTV-P, mouse mammary tumor virus basal promoter; SV40 polyA, SV40 virus polyadenylation signal; M5 and P3, PCR primers used to amplify inserts in the MCS; T7 and SP6, promoters that transcribe DNA sequences in sense orientation and antisense orientation, respectively. FseI, SfiI and NotI are rare-cutting restriction enzyme sites. perature, cells were cultured in fresh medium. For calcium phosphate precipitation, $7.5 \mu \mathrm{g}$ total DNA and $5 \times$ $10^{5}$ cells were used for each $60 \mathrm{~mm}$ plate. Cells were exposed to DNA-calcium phosphate precipitates for $8 \mathrm{~h}$ and glycerol-shocked (15\% glycerol in 20 mM HEPES-K ${ }^{+}, \mathrm{pH}$ 7.2) at room temperature for $1 \mathrm{~min}$. Glycerol was removed by washing cells twice with PBS. Thereafter, cells were cultured in fresh medium, and IPTG was added where indicated. In all transfections, pSG5 (Stratagene, La Jolla, CA, USA) DNA was used as carrier to bring up the total DNA amount. For stable transfections, cells were split [1 to 5] into hygromycin B or G418-containing mediums two days after transfection and further selected for 3 weeks.

\section{Construction of pEpiLac}

An IPTG-inducible episomal expression vector, $\mathrm{pEpiLac1}$ (Figure 1B), was constructed from the episomal vector pREP4 (Invitrogen, Carlsbad, CA, USA) and the pLac1 vector (Figure 1A). pLac1 was derived from pX12 (15), with a multiple cloning site (MCS) that was modified by the insertion of a pair of oligonucleotides containing three rare-cutting restriction enzyme recognition sites: FseI, SfiI and NotI (Figure 1C). pLac1 was double-digested with AflIII and SacI and the cohesive ends were filled-in using Klenow DNA polymerase (Promega, Madison, WI, USA). pREP4 was digested with SalI followed by Klenow DNA polymerase treatment. The large fragment from pREP4 contains the machinery for DNA replication as well as EBNA 1 and EBV oriP. The small fragment from pLac1 contains the IPTG-inducible MMTV basal promoter, MCS and the SV40 polyadenylation signal. pEpiLac1 resulted from the ligation of these two fragments (Figure 1B). pEpiLac3 was derived from pEpiLac1 by modifying the MCS (Figure 1C). Hygromycin B is required for long-term maintenance of cells that are transfected with this episomal vector because episomal vectors do not integrate into host cell chromosomes; in the absence of selection, episomal vectors are lost from the cell populations at rates of close to $5 \%$ per generation $(19,20)$. 


\section{Extraction of Episomal DNAs}

Episomal DNAs were isolated from MCF7/LAP5 cells using Hirt's extraction method $(4,5)$ with some modifications. Briefly, $1-2 \times 10^{6}$ cells were harvested in TEN buffer (40 mM Tris-HCl, $\mathrm{pH} 7.5,1 \mathrm{mM}$ EDTA and $150 \mathrm{mM}$ $\mathrm{NaCl}$.) washed once with cold PBS and then resuspended in $0.36 \mathrm{~mL}$ ice-cold TE buffer (10 mM Tris-HCl, $\mathrm{pH} 7.2$, and $10 \mathrm{mM}$ EDTA). SDS was added to the cell suspension for a final concentration of $1 \%$ and incubated at room temperature for 10-20 min after gentle mixing. One-fifth volume of $5 \mathrm{M} \mathrm{NaCl}$ was then added, gently mixed and incubated at $4^{\circ} \mathrm{C}$ overnight. Samples were centrifuged at $15000 \times \mathrm{g}$ for $30 \mathrm{~min}$ at $4^{\circ} \mathrm{C}$. The supernatant was collected and extracted once each with phenol, phenol: chloroform (1:1), and chloroform:iso- amyl alcohol (24:1). Episomal DNA was precipitated with $0.3 \mathrm{M}$ sodium acetate and 2-3 volume of absolute alcohol and dissolved in distilled water.

\section{Protein Assays}

For Western blot analysis, cells were lysed in RIPA buffer $(150 \mathrm{mM} \mathrm{NaCl}$, $50 \mathrm{mM}$ Tris- $\mathrm{HCl}, \mathrm{pH}$ 8.0, $1 \%$ Noni$\operatorname{det}^{\mathrm{TM}}$ P-40 (Sigma, St Louis, MO, USA), $0.5 \%$ sodium deoxycholate, $0.1 \%$ SDS, $1 \mathrm{mM}$ PMSF, $1 \%$ aprotinin and $10 \mu \mathrm{g} / \mathrm{mL}$ leupeptin) on ice for 10 min, centrifuged at $15000 \times g$ for 15 $\min$ at $4^{\circ} \mathrm{C}$. Protein were quantified using $\mathrm{D} c$ protein assay reagents (Bio-Rad Laboratories), separated in 10\% SDSPAGE and electroblotted. Proteins were detected by enhanced chemiluminescence (ECL) (Amersham Pharmacia Biotech, Piscataway, NJ, USA) using

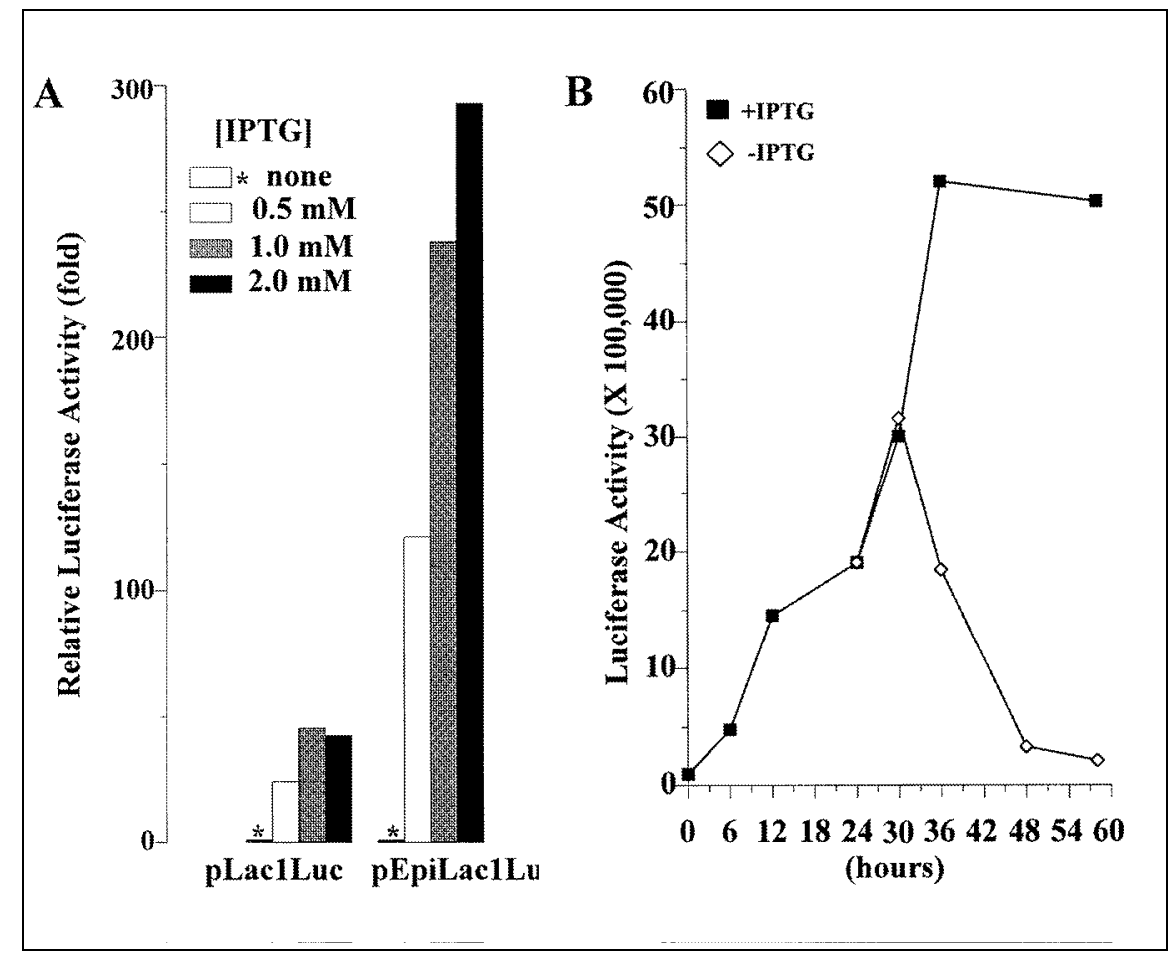

Figure 2. IPTG-induced expression of the luciferase reporter gene in MCF7/LAP5 cells. (A) MCF7/LAP5 cells were transiently cotransfected with pPGK $\beta$ Gal $(1 \mu \mathrm{g})$ together with either pLac1Luc $(2 \mu \mathrm{g})$ or pEpiLac1Luc ( $3 \mu \mathrm{g}$, about $25 \%$ less in molar amount than $2 \mu \mathrm{g} \mathrm{pLac} 1 \mathrm{Luc})$ using the calcium phosphate precipitation method. Luciferase activities from cell extracts were measured $32 \mathrm{~h}$ after IPTG stimulation and normalized with $\beta$-gal activities. The normalized luciferase activities from IPTG-treated cells were compared to those from the corresponding untreated cells, which were considered as 1 (indicated by ${ }^{*}$ ). (B) A stable population of MCF7/LAP5 cells transfected with pEpiLac1Luc was plated at $1 \times 10^{5}$ cells per $60 \mathrm{~mm}$ plate. One group of cells was continuously maintained in medium containing $1 \mathrm{mM}$ IPTG for indicated times. Another group of cells was incubated in medium containing IPTG for 24 $\mathrm{h}$ and washed twice with PBS and cultured in fresh medium without IPTG. At each time point, luciferase activities were measured and normalized with protein concentrations in whole cell extracts. Data were shown as representatives of three independent experiments. 
affinity-purified polyclonal antibodies, $\alpha$-Nur77 (7), $\alpha$-Cyr61 (13) and p27(C19) (Santa Cruz Biotechnology, Santa Cruz, CA, USA). Both luciferase and $\beta$-galactosidase ( $\beta$-gal) activities were measured using reagents from Promega (Madison, WI, USA) according to the manufacturer's instructions.

\section{RESULTS AND DISCUSSION}

\section{IPTG-Inducible Gene Expression in Human Breast Cancer Cells}

We have constructed the episomal vector pEpiLac (Figure 1B; see Materials and Methods), that combines features of the IPTG-inducible expression system and the episomal vector system. Inducible expression of exogenous genes cloned in this vector requires a cellular background that supplies the IPTG-dependent transactivator, LAP-

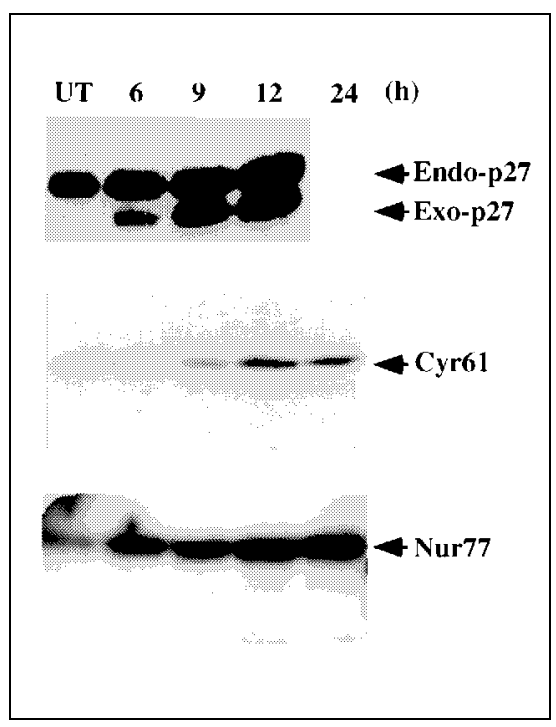

Figure 3. IPTG-induced expression of various exogenous genes in stable transfection assays. MCF7/LAP5 cells were transfected with $20 \mu \mathrm{g}$ pEpiLac1p27, pEpiLac3Nur77 or pEpiLac3Cyr61 by electroporation. Cells surviving hygromycin $\mathrm{B}$ selection were plated in $100 \mathrm{~mm}$ dishes $\left(1 \times 10^{6}\right.$ cells each) and stimulated with $1 \mathrm{mM}$ IPTG for indicated times or left untreated (UT). Cells were harvested in TEN buffer, washed twice with cold PBS and lysed in $50 \mu \mathrm{L}$ RIPA buffer. Ten microliters of cell lysates were resolved on $10 \%$ SDSPAGE. The expression of exogenous gene products was detected in Western blot analysis using affinity-purified polyclonal antibodies. Exogenous

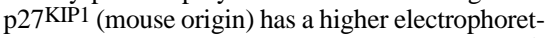
ic mobility than the endogenous human p $27^{\mathrm{KIP} 1}$, which served as internal control.
267 (3). We chose to establish an inducible system in the context of the human breast cancer cell line MCF7 because it is one of the most extensively studied cell lines in cancer biology. MCF7 cells were transfected with pX6LAP267 and pWLneo (5 $\mu \mathrm{g}$ and $0.5 \mu \mathrm{g}$ per $100 \mathrm{~mm}$ plate, respectively). pX6LAP267 is an autoregulatory vector that expresses LAP267 under an IPTG-inducible promoter, and thus a large amount of LAP267 is produced in the presence of IPTG (Pestov and Lau, unpublished data). pWLneo expresses the neomycin-resistant gene in a constitutive manner (Stratagene). After transfection, cells were selected against G418, and 15 independent, G418-resistant clonal cell lines were isolated.

The efficacy of these clonal cell lines (MCF7-LAP267 cells) to support IPTG-inducible gene expression was evaluated in transient co-transfection assays. These MCF7-LAP267 cells were co-transfected with either of two reporter constructs: pEpiLac1Luc, which expresses the luciferase gene under the IPTG-inducible promoter in the episomal pEpiLac1 vector (Figure 1B); or pLac1Luc, which expresses the luciferase gene under the corresponding promoter in the pLac1 plasmid vector (Figure 1A). These vectors were cotransfected with pPGK $\beta \mathrm{Gal}$, the $\beta$-gal expression vector, which serves as an internal control for transfection efficiencies (1). One clonal line, MCF7/ LAP5, showed a high level of IPTG-inducible luciferase activity and was further characterized (Figure 2A).

By contrast, the parental MCF7 cells showed nearly undetectable levels of luciferase activity with or without IPTG treatment (data not shown). Where luciferase activity was induced in pLac1Luc up to about 50-fold under the optimal IPTG concentration, the expression of luciferase was inducible up to 300 -fold under similar conditions when expressed using the episomal pEpiLac1Luc (Figure 2A). Thus, the episomal vector pEpiLac1 both maintains a low level of basal expression under uninduced conditions and allows a higher level of IPTG-inducible expression when compared to the non-episomal vector $\mathrm{pLac} 1$. We have also established the episomal inducible system in the human glioblastoma cell line U-373 and the human osteogenic sarcoma cell line U2OS, and similar results were obtained (unpublished data).

\section{On-Off Regulation of Gene Expression}

Because expression of exogenous genes in the pEpiLac vector can be turned on efficiently by IPTG induction, we examined whether this expression can be turned off following the removal of the inducer. We generated a population of MCF7/LAP5 cells stably transfected with pEpiLac1Luc and treated these cells with $1 \mathrm{mM}$ IPTG to induce the expression of luciferase (Figure 2B). After $24 \mathrm{~h}$ of treatment, the cells were either allowed to continue incubation in the presence of IPTG or washed twice with PBS and cultured in fresh medium without IPTG. After IPTG removal, the luciferase activities remained high for about $6 \mathrm{~h}$ and then quickly dropped to near basal levels 24 $\mathrm{h}$ thereafter (Figure 2B). However, without removal of IPTG, the luciferase activities continued to accumulate until a plateau was reached (Figure 2B). Thus, expression of the luciferase gene from pEpiLac1 was dependent on the presence of IPTG, and this induction was reversible.

\section{IPTG-Regulated Expression of Exogenous Genes in Stable Transfectants}

Exogenous luciferase reporter gene was efficiently expressed in both transient and stable transfection assays in MCF7/LAP5 cells (Figure 2). Next, we generated populations of MCF7/LAP5 cells stably transfected with pEpiLac vectors expressing either the p27KIP1 CDK inhibitor (18), the orphan nuclear receptor Nur77 (6) or the angiogenic inducer Cyr61 (2). Cells that survived hygromycin B selection was treated with $1 \mathrm{mM}$ IPTG for various durations. The expression of exogenous gene products was detected in Western blot analysis using affinity-purified polyclonal antibodies (Figure 3). Exogenous proteins was detectable as early as $6 \mathrm{~h}$ after IPTG treatment (Figure 3). Overexpression of p27KIP1 dramatically inhibited cell proliferation rate; cell growth was restored after the removal 
of IPTG inducer (unpublished data). This is consistent with the growth inhibitory effect of the p27 KIP1 (18). Without IPTG stimulation, exogenous p27KIP1 expression was undetectable (Figure 3), which indicates there was no detectable leakage in this system. Exogenous Cyr61 or Nur77 protein levels in cells not treated with IPTG were similar to the basal level of endogenous proteins (data not shown). The low or undetectable level of leakage in this system allows the regulated expression of growth inhibitory genes such as p $27^{\mathrm{KIP} 1}$ and suggests that this system may be useful for cytotoxic genes.

As is the case with other episomal vectors, the pEpiLac DNA can be maintained extra-chromosomally. When cells transfected with the $\mathrm{p} 27^{\mathrm{KIP} 1}$ construct were maintained in media containing hygromycin B for 10 months, episomal DNAs could still be easily harvested using Hirt's extraction (9). After amplification in bacterial cells, the integrity of these episomal DNAs was confirmed by restriction enzyme digestion and PCR analysis (data not shown). Therefore, this system can be applied in genetic selection procedures that require regulated on-off gene expression and recovery of transfected sequences, such as selections using genetic suppressor elements or selectable expression of transient growth arrest phenotype $(10,17)$.

\section{ACKNOWLEDGMENTS}

We would like to thank Dr. D.G. Pestov for sharing reagents and our colleagues for critical discussions. This work was supported National Institutes of Health Grant No. CA52220 and the Department of Defense.

\section{REFERENCES}

1.Adra, C.N., P.H. Boer and M.W. McBurney. 1987. Cloning and expression of the mouse pgk-1 gene and the nucleotide sequence of its promoter. Gene 60:65-74.

2.Babic, A.M., M.L. Kireeva, T.V. Kolesnikova and L.F. Lau. 1998. CYR61, a product of a growth factor-inducible immediate early gene, promotes angiogenesis and tumor growth. Proc. Natl. Acad. Sci. USA 95:63556360.

3.Baim, S.B., M.A. Labow, A.J. Levine and T.
Shenk. 1991. A chimeric mammalian transactivator based on the lac repressor that is regulated by temperature and isopropyl-D-thiogalactopyranoside. Proc. Natl. Acad. Sci. USA 88:5072-5076.

4.Bowers, M.T., S. Ramachandran and B.W. Yu. 1999. Modified miniprep method for the rapid recovery of episomes from transfected breast epithelial cells. BioTechniques 26:276282.

5.Brickell, P.M. and M.S. Patel. 1995. EpsteinBarr virus replication studies and their application to vector design. Mol. Biotechnol. 3:199-205.

6.Davis, I.J. and L.F. Lau. 1994 Endocrine and neurogenic regulation of the orphan nuclear receptors Nur77 and Nurr-1 in the adrenal glands. Mol. Cell Biol. 14:3469-3483.

7.Hazel, T.G., R. Misra, I.J. Davis, M.E. Greenberg and L.F. Lau. 1991. Nur77 is differentially modified in PC12 cells upon membrane depolarization and growth factor treatment. Mol. Cell Biol. 11:3239-3246.

8.Heinzel, S.S., P.J. Krysan, M.P. Calos and R.B Dubridge. 1988. Use of simian virus 40 replication to amplify Epstein-Barr virus shuttle vectors in human cells. J. Virol. 62:37383746.

9.Hirt, B. 1967. Selective extraction of polyoma DNA from infected mouse cell cultures. J. Mol. Biol. 26:365-369.

10.Holzmayer, T.A., D.G. Pestov I.B. and Roninson. 1992. Isolation of dominant negative mutants and inhibitory antisense RNA sequences by expression selection of random DNA fragments. Nucleic Acids Res. 20:711717.

11.Jalanko, A., A. Kallio and I. Ulmanen. 1988. Comparison of mammalian cell expression vectors with and without an EBV-replicon. Arch. Virol. 103:157-166.

12.James, M.R., A. Stary, L. Daya-Grosjean, C. Drougard and A. Sarasin. 1989. Comparative study of Epstein-Barr virus and SV40based shuttle-expression vectors in human repair-deficient cells. Mutat. Res. 220:169185.

13.Kireeva, M.L., F.E. MO, G.P. Yang and L.F. Lau. 1996. Cyr61, a product of a growth factor-inducible immediate-early gene, promotes cell proliferation, migration, and adhesion. Mol. Cell Biol. 16:1326-1334.

14.Margolskee, R.F., P. Kavathas and P. Berg. 1988. Epstein-Barr shuttle vector for stable episomal replication of cDNA expression libraries in human cells. Mol. Cell. Biol. 8:2837-2847.

15.Pestov, D.G. 1997. Genetic selection of growth-inhibitory sequences in mammalian cells. PhD thesis, University of Illinois at Chicago.

16.Pestov, D.G., T.M. Grzeszkiewicz and L.F. Lau. 1998. Isolation of growth suppressors from a cDNA expression library. Oncogene 17:3187-3197.

17.Pestov, D.G. and L.F. Lau. 1994. Genetic selection of growth-inhibitory sequences in mammalian cells. Proc. Natl. Acad. Sci. USA 91:12549-12553.

18.Polyak, K., M.H. Lee, H. Erdjument-Bromage, A. Koff, J.M. Roberts, P. Tempst and J. Massague. 1994. Cloning of p27Kip1, a cy- clin-dependent kinase inhibitor and a potential mediator of extracellular antimitogenic signals. Cell 78:59-66.

19.Yates, J.L. and N. Guan. 1981. Epstein Barr virus-derived Plasmids replicate only once per cell cycle and are not amplified after entry into cells. J. Virol. 65:483-488.

20.Yates, J.L., N. Warren and B. Sugden. 1985. Stable replication of plasmids derived from Epstein-Barr virus in various mammalian cells. Nature 313:812-815.

Received 28 June 1999; accepted 12 October 1999.

\section{Address correspondence to:}

Dr. Lester F. Lau

Department of Molecular Genetics (M/C 669)

University of Illinois College of Medicine

900 South Ashland Avenue

Chicago, IL 60607-7170, USA

Internet: lflau@uic.edu 